\title{
Acute porcine reproductive and respiratory syndrome outbreaks in immunized sow herds: from occurrence to stabilization under whole herd vaccination strategy
}

\author{
Sung Ho Moon ${ }^{1,2 \uparrow}$, Sung J. Yoo ${ }^{1, \uparrow}$, Sang Hyun Noh ${ }^{1}$, Taeyong Kwon ${ }^{1}$, Dong Uk Lee ${ }^{1}$, Sang H. Je${ }^{1}$, \\ Myung Hyee Kim ${ }^{1,3}$, Sang Won Seo ${ }^{1,3}$, Young S. Lyoo ${ }^{1, *}$ \\ ${ }^{1}$ College of Veterinary Medicine, Konkuk University, Seoul 05029, Korea \\ ${ }^{2}$ Breeding Pig Improvement Center, National Agricultural Cooperative Federation, Yeounggwang-gun 57053, Korea \\ ${ }^{3}$ Hipra Korea, Inc., Seongam 13557, Korea
}

(Received: January 30, 2018; Revised: May 23, 2018; Accepted: May 30, 2018)

\begin{abstract}
Outbreaks of porcine reproductive and respiratory syndrome virus (PRRSV) in vaccinated sow herds from occurrence to stabilization were monitored and analyzed in terms of serology and reproductive performance. Three different conventional pig farms experienced severe reproductive failures with the introduction of a type 1 PRRSV. These farms had adopted mass vaccination of sows using a type 2 PRRSV modified live vaccine (MLV). Therefore, to control the type 1 PRRSV, an alternative vaccination program utilizing both type 1 and type 2 MLV was undertaken. Following whole herd vaccinations with both types of MLV, successful stabilization of PRRS outbreaks was identified based on serological data (no viremia and downward trends in ELISA antibody titers in both sows and suckling piglets) and recovery of reproductive performance. Additionally, through comparison of the reproductive parameters between outbreak and non-outbreak periods, it was identified that PRRSV significantly affected the farrowing rate and the number of suckling piglets per litter at all three pig farms. Comparison of reproductive parameters between periods when the different vaccination strategies were applied revealed that the number of piglets born in total and born dead per litter were significantly increased after the introduction of the type 1 PRRS MLV.
\end{abstract}

Keywords: disease outbreaks, herd immunity, heterologous effects of vaccine, porcine reproductive and respiratory syndrome virus, reproductive failures

\section{Introduction}

Porcine reproductive and respiratory syndrome virus (PRRSV) has been recognized as one of major pathogens responsible for tremendous economic losses in the swine industry [22]. There are two main genotypes of PRRSV: genotype 1 (European) and genotype 2 (North American) [7, 28]. Many Asian countries including Korea harbor both genotypes of PRRSV $[5,12,15,26]$. In Korea, the presence of PRRSV genotype 2 was reported in 1994, followed by that of PRRSV genotype 1 in 2006 and both genotypes have been found circulating concurrently in swine herds $[6,14,17,20]$.

Many strategies have been implemented to control and eradicate PRRSV, including management strategies (early weaning, herd closure, acclimatization, and all-in-all-out replacement system), improvement of biosecurity, and vaccination. Of these measures, vaccination is recognized as a common and popular method owing to its low cost and wide application $[8,21]$. When combined with other measures, vaccination can be an effective tool for both PRRSV stabilization and eradication [2, 4].

However, limited or partial cross protection among heterologous PRRSV strains has been demonstrated by many other research groups, which underscores the importance of antigenic match between vaccines and circulating virus strains [3, 16, 19, 27]. In this study, we report acute PRRSV outbreaks in vaccinated sow herds, which were provoked by limited cross protection against heterologous PRRSV strains and those stabilization with a proper vaccination strategy. Also, we provide the case study analyses of how the sow performance and serological data had been changed during the disease outbreaks and stabilization.

\footnotetext{
*Corresponding author

Tel: +82-2-450-3719, Fax: +82-2-6008-3791

E-mail: lyoo@konkuk.ac.kr

The first two authors contributed equally to this work.
} 


\section{Materials and Methods}

All animals were reared and handled with due regard for their welfare. Three breeding pig farms (Farms A, B, and C) were certified by Hazard Analysis Critical Control Point (HACCP) for sanitation safety in the livestock rearing stage.

The trial was performed at three different farrow-to-feeder farms with 530, 980, and 980 sows located in the same province. These sows had been diagnosed as type 2 PRRSV-positive, and MLV vaccination against the type 2 virus (Ingelvac PRRS MLV; Boehringer Ingelheim Animal Health, USA) had been carried out accordingly over 4 years. However, unexpectedly, the swine herds had experienced an acute PRRS outbreak characterized by mid to late term abortions (usually after 40-day gestation period) with a newly introduced type 1 PRRSV strain (Fig. 1). As a measure of intervention, a type 1 PRRS vaccine (Unistrain PRRS; HIPRA, Spain) was additionally administered to the sows while maintaining vaccination against type 2 PRRSV.

Two different commercial PRRS vaccines were administered intramuscularly to the sows according to the manufacturer's instructions. Quarterly vaccination was performed regardless of pregnancy status; single use of type 2 PRRS vaccine before the abortion storms and administrations of both type 1 and 2 PRRS vaccines at one-month interval, except for the first two consecutive uses of type 1 PRRS MLV, after the outbreak occurrence (Fig. 1A). Blood samples were collected from the sows and suckling pigs one month before administration of type $1 \mathrm{MLV}, 2$ months post the introduction of type 1 PRRS vaccine $\left(2 \mathrm{mpv}_{1}\right), 7 \mathrm{mpv}_{1}$ and $13 \mathrm{mpv}_{1}$ for further analyses (Fig. 1A). Thirty serum samples (20 from sows and 10 from suckling piglets) per each pig farm were collected at each time point.

For determination of the causative agent for abortion, polymerase chain reaction (PCR) was performed for PRRSV, porcine circovirus type 2, classical swine fever virus, porcine parvovirus, Aujeszky's disease virus, Japanese encephalitis virus, and encephalomyocarditis virus according to the method as described previously $[13,18,30]$. Additionally, to obtain PRRSV sequences, the complete region of open reading frame 5 was amplified following the published method [29]. The obtained PCR products were cloned into the pGEM-T easy vector (Promega, USA) and propagated in Escherichia coli DH5á cells. The plasmids were then extracted and used for sequencing (Macrogen, Korea). For the phylogenetic analyses, a neighbor-joining tree was constructed using the MEGA7 software with 1,000 bootstrap replicates.

For the titration of antibodies against PRRSV per individual serum sample, IDEXX PRRS HerdChek X3 Kit (IDEXX Laboratories, USA) was utilized. The enzyme-linked immunosorbent assay (ELISA) targeting anti-PRRSV nucleocapsid antibodies was performed according to the manufacturer's instructions (positive: sample/positive $(\mathrm{S} / \mathrm{P})$ value $\geq 0.4$; negative: $\mathrm{S} / \mathrm{P}$ value $<0.4$ ).

The reproductive performance was evaluated on the basis
A

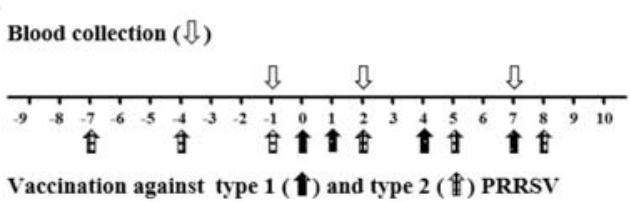

B

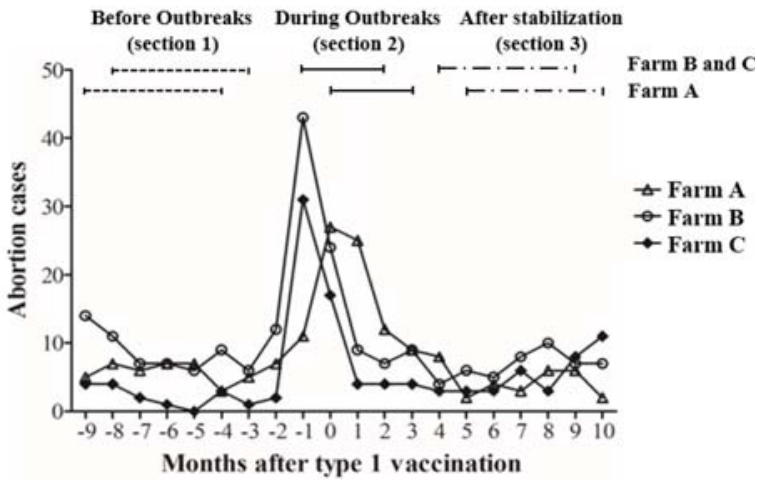

Fig. 1. Experimental schedule for vaccination and blood collection (A) and the changes in the number of abortion cases with the time course of porcine reproductive and respiratory syndrome (PRRS) outbreaks (B). The starting point of type 1 vaccination was marked as zero and the abortion cases were recorded monthly per farm. The arrows in (A) indicate the time point at which blood collection (open arrows) and vaccination against type 1 (closed arrows) and type 2 porcine reproductive and respiratory syndrome virus (PRRSV) (arrows with plaid patterns) were performed. The lines with unique styles (solid, dotted, or dash-dot) in (B) represent the periods of data collection for comparative analysis according to the progress of PRRS outbreaks.

of fertility traits (pregnancy rate, farrowing rate, abortion cases, weaning-to-estrus interval, and removal of sows) and litter traits (total number of piglets born per litter, number of piglets born dead per litter, number of suckling and weanling piglets per litter, and percentage of death losses of suckling pigs). The investigation period for a total of 20 month-points was subdivided into three sections; Section 1 (before outbreaks, 6 month-points), Section 2 (during outbreaks, 4 monthpoints), and Section 3 (after stabilization, 6 month-points) (Fig. 1B). The reproductive parameters of three sections were compared by following orders; Firstly, to identify changes in the evaluated parameters by the alteration of vaccination strategy, those of Section 1 and 3 were compared. Secondly, to determine significantly affected reproductive parameter during an acute PRRS outbreak, those of Section 2 and Section 1 and 3 were compared. The start point of the disease outbreaks (Section 2) was set to the month of the highest abortion cases and the duration of Section 2 to three months (4 month-points) since the effects of abortions are reflected afterward on the other parameter estimates, especially for litter traits. Further, to minimize the possible influences by seasonal condition, the sections of before outbreaks and after 
Table 1. PRRSV antigen detection in the serum samples of sows and suckling pigs

\begin{tabular}{|c|c|c|c|c|c|}
\hline \multirow{2}{*}{ Period } & \multirow{2}{*}{ Section } & \multirow{2}{*}{ Type } & \multicolumn{3}{|c|}{ Experimental farms } \\
\hline & & & $\mathrm{A}$ & $\mathrm{B}$ & $\mathrm{C}$ \\
\hline \multirow{4}{*}{$-1 m p v_{1}$} & Sows $(n=60)$ & 1 & + & + & + \\
\hline & & 2 & - & - & - \\
\hline & Suckling pigs $(\mathrm{n}=30)$ & 1 & - & + & + \\
\hline & & 2 & $+^{*}$ & - & - \\
\hline \multirow[t]{4}{*}{$2 \mathrm{mpv}_{1}$} & Sows $(n=60)$ & 1 & - & - & - \\
\hline & & 2 & - & - & - \\
\hline & Suckling pigs $(\mathrm{n}=30)$ & 1 & + & $+^{*}$ & - \\
\hline & & 2 & - & $+*$ & - \\
\hline \multirow[t]{4}{*}{$7 \mathrm{mpv}_{1}$} & Sows $(n=60)$ & 1 & - & - & - \\
\hline & & 2 & - & - & - \\
\hline & Suckling pigs $(\mathrm{n}=30)$ & 1 & - & - & - \\
\hline & & 2 & - & - & - \\
\hline \multirow[t]{4}{*}{$13 \mathrm{mpv}_{1}$} & Sows $(n=60)$ & 1 & - & - & - \\
\hline & & 2 & - & - & - \\
\hline & Suckling pigs $(\mathrm{n}=30)$ & 1 & - & - & - \\
\hline & & 2 & - & - & - \\
\hline
\end{tabular}

*The PRRSVs were identified as vaccine strains that were used for sow immunization. $\mathrm{mpv}_{1}$, months post the type 1 PRRS vaccine introduction.

stabilization were constructed temporally symmetrical with 12-month interval.

SPSS (ver. 21; SPSS, USA) was used for statistical analysis. For the comparison of reproductive parameters, student $t$ test and Mann-Whitney test were selectively used according to the group normality. Statistical significance of $p$ value was set at 0.05 .

\section{Results}

PCR results indicated that serum samples were positive only for PRRSV among several tested pathogens associated with abortion. In all three pig farms, viremia caused by type 1 and type 2 PRRSV strains was confirmed in both serum samples of suckling piglets and sows at $-1 \mathrm{mpv}_{1}$ and $2 \mathrm{mpv}_{1}$ (Table 1). A total of nine PRRSV strains were detected in this study; seven strains were type 1 and the other two strains were type 2 . All the type 2 strains $(n=2)$ and a type 1 strain $(n=1)$ clustered with the vaccine strains, sharing over $98 \%$ genetic homology, while the other type 1 strains $(n=6)$ formed a distinct phylogenetic cluster from the group that contains type 1 PRRS vaccine strain, exhibiting $12.8-13.3 \%$ genetic differences (Fig. 2). Viremia caused by the field strains of type 1 PRRSV was not detected further in all tested serum samples collected since $2 \mathrm{mpv}_{1}$, except for samples from suckling piglets of Farm A collected at $2 \mathrm{mpv}_{1}$ (Table 1). The PRRSV strain was identified to have genetic identity over 99.5\% in ORF5 nucleotide sequences with the previously diagnosed one in Farm A.

The mean titer of anti PRRSV antibodies was highest in sow serum samples collected one month before the use of the type 1 PRRS vaccine $\left(-1 \mathrm{mpv}_{1}\right)$ but gradual downward trends

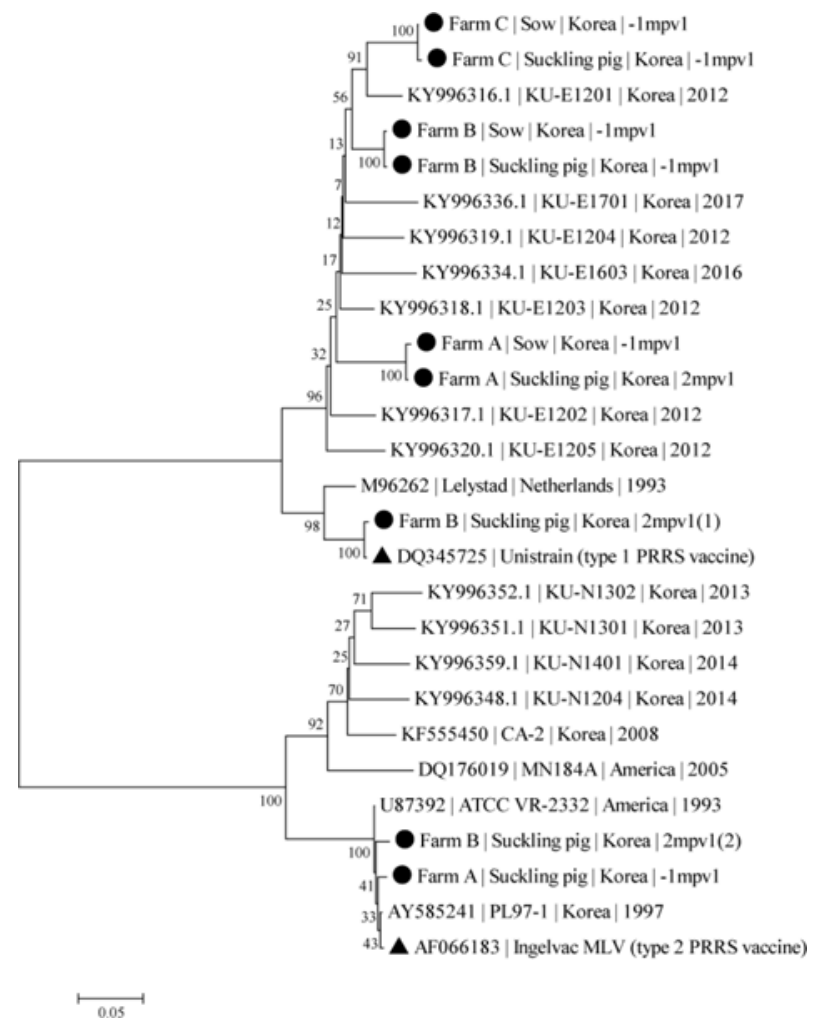

Fig. 2. Phylogenetic relationships between detected strains of types 1 and 2 PRRSV and vaccine strains used in this study. The phylogenetic tree was generated based on ORF5 nucleotide sequences using the neighbor-joining method. The reliability of the tree was assessed by bootstrap analysis of 1,000 replications. The detected PRRSVs were marked by a black circle with the information on farm, host, and sampling time. The PRRS vaccine strains used in this study were indicated by a black triangle. 

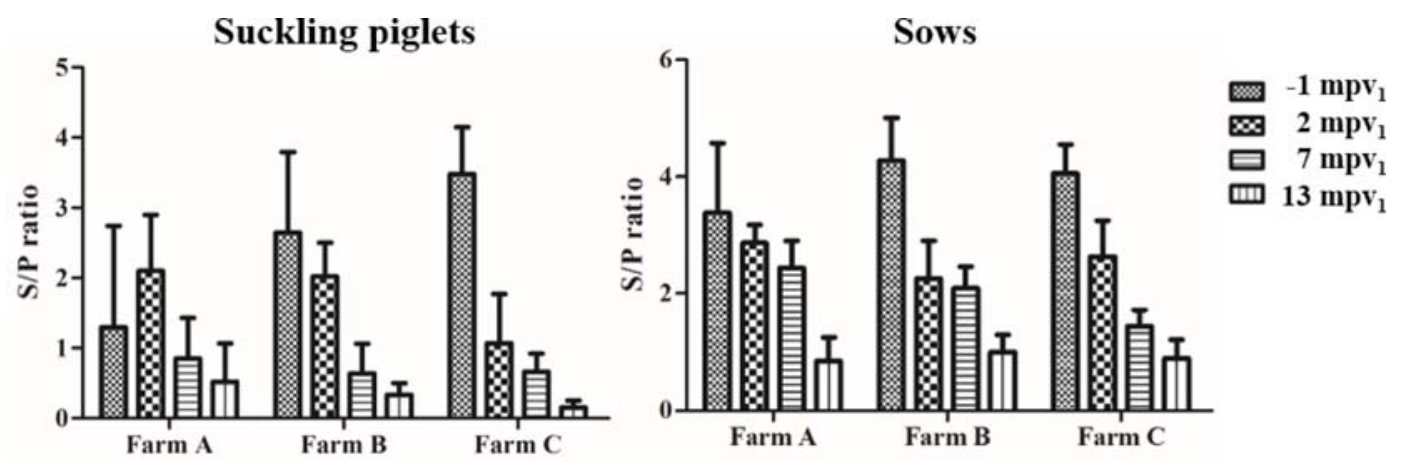

Fig. 3. Mean titers of anti-PRRSV antibodies for serum samples from three farms collected at one month before $\left(-1 \mathrm{mpv}_{1}\right)$, two months $\left(2 \mathrm{mpv}_{1}\right)$, seven months $\left(7 \mathrm{mpv}_{1}\right)$, and thirteen months $\left(13 \mathrm{mpv}_{1}\right)$ after type $1 \mathrm{PRRS}$ vaccination. $\mathrm{S} / \mathrm{P}$ ratio, sample/positive ratio.

Table 2. Litter traits of three pig farms during PRRS outbreaks (mean \pm SD)

\begin{tabular}{|c|c|c|c|c|}
\hline Reproductive parameters & Farm & Before outbreaks & During outbreaks & After stabilization \\
\hline \multirow[t]{3}{*}{ Total born/litter ${ }^{\dagger}$} & $A^{*}$ & $12.2 \pm 0.33$ & $11.5 \pm 0.62$ & $12.8 \pm 0.57$ \\
\hline & $\mathrm{B}^{*}$ & $12.2 \pm 0.24$ & $12 \pm 0.27$ & $13.4 \pm 0.34$ \\
\hline & $\mathrm{C}^{*}$ & $11.7 \pm 0.17$ & $11.6 \pm 0.24$ & $12.5 \pm 0.23$ \\
\hline \multirow[t]{3}{*}{ Dead born/litter ${ }^{\dagger}$} & $A^{*}$ & $1.6 \pm 0.23$ & $1.8 \pm 0.19$ & $2.2 \pm 0.29$ \\
\hline & $\mathrm{B}^{*}$ & $1.7 \pm 0.14$ & $2.5 \pm 0.74$ & $2.6 \pm 0.22$ \\
\hline & $\mathrm{C}^{*}$ & $1.2 \pm 0.04$ & $1.8 \pm 0.31$ & $1.7 \pm 0.21$ \\
\hline \multirow[t]{3}{*}{ Suckling pigs/litter } & $A^{*}$ & $10.6 \pm 0.31$ & $9.7 \pm 0.66$ & $10.6 \pm 0.35$ \\
\hline & $\mathrm{B}^{*}$ & $10.6 \pm 0.13$ & $9.5 \pm 0.5$ & $10.9 \pm 0.28$ \\
\hline & $\mathrm{C}^{*}$ & $10.9 \pm 0.24$ & $9.8 \pm 0.31$ & $10.8 \pm 0.18$ \\
\hline \multirow[t]{3}{*}{ Weanling pigs/litter } & A & $10.2 \pm 0.26$ & $9.5 \pm 1.3$ & $10.2 \pm 0.62$ \\
\hline & $\mathrm{B}^{*}$ & $10.4 \pm 0.36$ & $9.3 \pm 0.84$ & $10.5 \pm 0.45$ \\
\hline & $\mathrm{C}^{*}$ & $10.7 \pm 0.21$ & $9.7 \pm 0.69$ & $10.5 \pm 0.16$ \\
\hline \multirow[t]{3}{*}{ Death rate of suckling pigs ${ }^{\sharp}$} & $A^{*}$ & $2.9 \pm 0.83$ & $5.3 \pm 2.73$ & $2.8 \pm 0.55$ \\
\hline & $\mathrm{B}^{*}$ & $2.3 \pm 0.26$ & $5.5 \pm 1.1$ & $2.2 \pm 0.81$ \\
\hline & $\mathrm{C}$ & $2.2 \pm 0.45$ & $2.7 \pm 0.8$ & $2.4 \pm 0.53$ \\
\hline
\end{tabular}

*Farm showing significant changes in the corresponding parameter when compared by criteria such as vaccine strategy or disease outbreak. 'Significantly affected reproductive parameter in all three different farms by the alteration of vaccine strategy (Section $1 v s$. Section 3$)(p<$

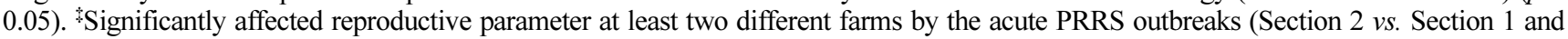
3) $(p<0.05)$.

of antibody titer over time was observed in all three farms. Likewise, in suckling pigs, similar pattern of antibody titer changes was detected except for in Farm A, in which serum antibody titer was highest at $2 \mathrm{mpv}_{1}$ (Fig. 3). Except for suckling pigs of Farm A, the mean antibody titers of sows and suckling pigs at $13 \mathrm{mpv}_{1}$ dropped to below 0.4 , the threshold for defining an antibody positive status.

Interestingly, when reproductive parameters between Section 1 and 3 of three farms were compared, significantly increased number of piglets born in total per litter was commonly identified also with comparable increases in piglets born dead per litter, which resulted in similar productivity in the number of suckling piglets per litter (Table 2).

A comparison analysis of reproductive parameters between outbreak period (Section 2) and non-outbreak periods (Sec- tion 1 and 3) revealed that PRRSV outbreaks had affected farrowing rate and the number of suckling piglets per litter at a significantly level in all three pig farms. Additionally, substantial increase in abortion cases and death rates of suckling pigs and decrease in pregnancy rate and the number of weanling pig per litter were identified in at two pig farms (Tables 2 and 3$)$.

\section{Discussion}

The economic losses caused by PRRSV has been increasing in many countries and the increased portion of breeding herd compared with growing pig herd is remarkable, which highlights the importance of PRRSV control in sows [22]. Currently, MLV vaccination is considered as one of the effec- 
Table 3. Fertility traits of three pig farms during PRRS outbreaks (mean \pm SD)

\begin{tabular}{|c|c|c|c|c|}
\hline Reproductive parameters & Farm & Before outbreaks & During outbreaks & After stabilization \\
\hline \multirow[t]{3}{*}{ Pregnancy rate ${ }^{\dagger}(\%)$} & A & $87.3 \pm 3.33$ & $89.8 \pm 1.05$ & $86.0 \pm 5.35$ \\
\hline & $\mathrm{B}^{*}$ & $86.5 \pm 3.10$ & $83.2 \pm 2.71$ & $89.4 \pm 1.83$ \\
\hline & $\mathrm{C}^{*}$ & $90.7 \pm 2.05$ & $87.5 \pm 4.75$ & $91.4 \pm 1.15$ \\
\hline \multirow[t]{3}{*}{ Farrowing $\operatorname{rate}^{\dagger}(\%)$} & $A^{*}$ & $82.0 \pm 1.94$ & $72.4 \pm 5.56$ & $82.4 \pm 4.46$ \\
\hline & $\mathrm{B}^{*}$ & $81.5 \pm 3.29$ & $71.3 \pm 2.07$ & $82.9 \pm 1.62$ \\
\hline & $\mathrm{C}^{*}$ & $84.9 \pm 1.66$ & $78.3 \pm 4.09$ & $86.3 \pm 3.08$ \\
\hline \multirow[t]{3}{*}{ Removals (\%) } & A & $5.0 \pm 2.20$ & $3.3 \pm 1.35$ & $4.0 \pm 0.77$ \\
\hline & $\mathrm{B}$ & $4.0 \pm 0.44$ & $3.9 \pm 0.76$ & $4.1 \pm 0.92$ \\
\hline & $\mathrm{C}$ & $4.0 \pm 0.44$ & $3.3 \pm 1.29$ & $4.7 \pm 0.93$ \\
\hline \multirow[t]{3}{*}{ Weaning to estrus interval (d) } & A & $6.6 \pm 0.66$ & $7.3 \pm 0.44$ & $7.1 \pm 1.04$ \\
\hline & B & $8.3 \pm 1.66$ & $6.4 \pm 0.70$ & $7.4 \pm 1.18$ \\
\hline & $\mathrm{C}$ & $8.3 \pm 1.66$ & $6.4 \pm 0.70$ & $7.4 \pm 1.18$ \\
\hline
\end{tabular}

*Farm showing significant changes in the corresponding parameter when compared by criteria such as vaccine strategy or disease outbreak. †'Significantly affected reproductive parameter at least two different farms by the acute PRRS outbreaks (Section 2 vs. Section 1 and 3$)(p<$ $0.05)$.

tive measures against PRRSV infection [21]. We had tried to control and stabilize unexpected type 1 PRRSV outbreaks in vaccinated breeding herds via altered vaccination strategy using both type 1 and 2 PRRS vaccines. In this study, we report analyses of the field cases in perspective of reproductive performance and serology.

Downward trends in antibody titers against PRRSV were observed in sows and suckling pigs of three pig farms over time after acute PRRS outbreaks, which indicates stabilization against the field strains of PRRSV. The continuous decrease in antibody titers despite the repetitive vaccination can be attributed to the low replication level of vaccine strains within host $[24,25]$. In the context of replication efficiency, higher antibody concentration in serum samples of suckling piglets of Farm A collected at $2 \mathrm{mpv}_{1}$ than that collected at $7 \mathrm{mpv}_{1}$ can be explained by the presence of the field PRRSV strain.

Disappearance of PRRSV antigen and no rebound of antibody titers against PRRSV in serum samples of sows and suckling piglets until $13 \mathrm{mpv}_{1}$ proved no more active virus circulation. Although the number of tested samples was not sufficient to reflect the disease status of whole-herd, reproductive performance recovered from PRRS outbreaks without sign of further exacerbation indirectly supports the stabilization of PRRSV at the farm level. The concept of "herd immunity" has been regarded instrumental in the control and eradication of infectious disease. Highly immunized group can effectively limit disease circulation and thereby reduce the chance of non-immunized individuals to be infected [11]. In these PRRS outbreaks, we had tried to build herd immunity against PRRSV via mass vaccination and succeeded the stabilization of PRRSV with recovered reproductive performance.

Several studies have reported about the influences of MLV vaccination on sows according to the pregnancy state with conflicting results $[1,9,10,23]$. For example, according to one research group, PRRS-MLV vaccination (Ingelvac PRRS MLV; Boehringer Ingelheim Animal Health) of sows during the gestation period reduced their reproductive performance, resulting in a decreased number of pigs born alive and weaned and an increased number of stillborn and mummified fetuses [10]. Conversely, other research group reported that PRRSMLV vaccination (Porcilis PRRS; MSD Animal Health, USA) of sows at any stage of gestation conferred protection against PRRSV without any adverse effect [1]. These opposite results could be related to differences in vaccine strains used for each investigation. In this view point, different vaccine strain usage between periods before and after PRRS outbreaks cannot be excluded as a contributing factor for the significant changes in the number of piglets born in total and born dead per litter. Further investigation of sow safety as per PRRS vaccine strain seems to be needed.

As described in Table 2 and 3, several reproductive parameters were found to be significantly affected by PRRS outbreaks under field conditions. Specifically, notable reduction in farrowing rate and the number of suckling piglets per litter after PRRS outbreaks was common in three pig farms, which represents that these two clinical parameters can be sensitive criteria estimating the reproductive performance during or after disease outbreaks.

In this study, we identified that the type 2 PRRS vaccine cannot confer cross protection against type 1 PRRSV infection under field conditions through the observations of abruptly increased abortion cases. Accordingly, we examined the immunization strategy of using both type 1 and type 2 PRRS vaccines in alternate manner following acute PRRS outbreaks and succeeded in the stabilization of PRRSV with no evidences of adverse effect or virus circulation. Further, we specified reproductive parameters significantly influenced by the altered vaccination regimen and PRRS outbreak. These 
findings described in this case study will be a valuable information for swine practitioners and producers.

\section{References}

1. Alexopoulos C, Kritas SK, Kyriakis CS, Tzika E, Kyriakis SC. Sow performance in an endemically porcine reproductive and respiratory syndrome (PRRS)-infected farm after sow vaccination with an attenuated PRRS vaccine. Vet Microbiol 2005, 111, 151-157.

2. Berton P, Normand V, Martineau GP, Bouchet F, Lebret A, Waret-Szkuta A. Evaluation of porcine reproductive and respiratory syndrome stabilization protocols in 23 French Farrow-to-finish farms located in a high-density swine area. Porcine Health Manag 2017, 3, 11.

3. Bonckaert $\mathbf{C}$, van der Meulen $\mathbf{K}$, Rodriguez-Ballarà I, Pedrazuela Sanz R, Martinez MF, Nauwynck HJ. Modifiedlive PRRSV subtype 1 vaccine UNISTRAIN ${ }^{\circledR}$ PRRS provides a partial clinical and virological protection upon challenge with East European subtype 3 PRRSV strain Lena. Porcine Health Manag 2016, 2, 12.

4. Charerntantanakul W. Porcine reproductive and respiratory syndrome virus vaccines: immunogenicity, efficacy and safety aspects. World J Virol 2012, 1, 23-30.

5. Chen N, Cao Z, Yu X, Deng X, Zhao T, Wang L, Liu Q, Li X, Tian K. Emergence of novel European genotype porcine reproductive and respiratory syndrome virus in mainland China. J Gen Virol 2011, 92, 880-892.

6. Choi EJ, Lee CH, Song JY, Song HJ, Park CK, Kim B, Shin YK. Genetic diversity of porcine reproductive and respiratory syndrome virus in Korea. J Vet Sci 2013, 14, 115-124.

7. Collins JE, Benfield DA, Christianson WT, Harris L, Hennings JC, Shaw DP, Goyal SM, McCullough S, Morrison RB, Joo HS, Gorcyca D, Chladek D. Isolation of swine infertility and respiratory syndrome virus (isolate ATCC VR-2332) in North America and experimental reproduction of the disease in gnotobiotic pigs. J Vet Diagn Invest 1992, 4, 117-126.

8. Corzo CA, Mondaca E, Wayne S, Torremorell M, Dee S, Davies P, Morrison RB. Control and elimination of porcine reproductive and respiratory syndrome virus. Virus Res 2010, 154, 185-192.

9. Dewey CE, Wilson S, Buck P, Leyenaar JK. Effects of porcine reproductive and respiratory syndrome vaccination in breeding-age animals. Prev Vet Med 2004, 62, 299-307.

10. Dewey CE, Wilson S, Buck P, Leyenaar JK. The reproductive performance of sows after PRRS vaccination depends on stage of gestation. Prev Vet Med 1999, 40, 233241.

11. Fine P, Eames K, Heymann DL. "Herd immunity": a rough guide. Clin Infect Dis 2011, 52, 911-916.

12. Iseki H, Takagi M, Kawashima K, Shibahara T, Kuroda Y, Tsunemitsu H, Yamakawa M. Pathogenicity of emerging Japanese type 1 porcine reproductive and respiratory syndrome virus in experimentally infected pigs. J Vet Med Sci 2016, 77, 1663-1666.

13. Je SH, Kwon T, Yoo SJ, Lee DU, Lee S, Richt JA, Lyoo YS. Classical swine fever outbreak after modified live LOM strain vaccination in naive pigs, South Korea. Emerg Infect
Dis 2018, 24, 798-800.

14. Kim JY, Lee SY, Sur JH, Lyoo YS. Serological and genetic characterization of the European strain of the porcine reproductive and respiratory syndrome virus isolated in Korea. Korean J Vet Res 2006, 46, 363-370.

15. King SJ, Ooi PT, Phang LY, Allaudin ZNB, Loh WH, Tee CY, How SP, Yip LS, Choo PY, Lim BK. Phylogenetic characterization of genes encoding for viral envelope glycoprotein (ORF5) and nucleocapsid protein (ORF7) of porcine reproductive \& respiratory syndrome virus found in Malaysia in 2013 and 2014. BMC Vet Res 2017, 13, 3.

16. Ko S, Seo S, Sunwoo S, Yoo SJ, Kim M, Lyoo YS. Efficacy of commercial genotype 1 porcine reproductive and respiratory syndrome virus (PRRSV) vaccine against field isolate of genotype 2 PRRSV. Vet Immunol Immunopathol 2016, 172, 43-49.

17. Kweon C, Kwon B, Lee H, Cho J, Hwang E, Shin J, Yoon Y, Kang Y, An S, Kim Y, Huh W, Jun M, Wensvoort G. Isolation of porcine reproductive and respiratory syndrome virus (PRRSV) in Korea. Korean J Vet Res 1994, 34, 77-83.

18. Kwon T, Lee DU, Yoo SJ, Je SH, Shin JY, Lyoo YS. Genotypic diversity of porcine circovirus type 2 (PCV2) and genotype shift to PCV2d in Korean pig population. Virus Res 2017, 228, 24-29.

19. Labarque GG, Nauwynck HJ, van Woensel PAM, Visser N, Pensaert MB. Efficacy of an American and a European serotype PRRSV vaccine after challenge with American and European wild-type strains of the virus. Vet Res 2000, 31, 97.

20. Lee DU, Yoo SJ, Kwon T, Je SH, Shin JY, Byun JJ, Kim MH, Lyoo YS. Genetic diversity of ORF 4-6 of type 1 porcine reproductive and respiratory syndrome virus in naturally infected pigs. Vet Microbiol 2017, 199, 54-61.

21. Lyoo YS. Porcine reproductive and respiratory syndrome virus vaccine does not fit in classical vaccinology. Clin Exp Vaccine Res 2015, 4, 159-165.

22. Neumann EJ, Kliebenstein JB, Johnson CD, Mabry JW, Bush EJ, Seitzinger AH, Green AL, Zimmerman JJ. Assessment of the economic impact of porcine reproductive and respiratory syndrome on swine production in the United States. J Am Vet Med Assoc 2005, 227, 385-392.

23. Olanratmanee EO, Thanawongnuwech R, Kunavongkrit A, Tummaruk P. Reproductive performance of sows with and without PRRS modified live virus vaccination in PRRSvirus-seropositive herds. Trop Anim Health Prod 2014, 46, 1001-1007.

24. Opriessnig T, Halbur PG, Yoon KJ, Pogranichniy RM, Harmon KM, Evans R, Key KF, Pallares FJ, Thomas P, Meng XJ. Comparison of molecular and biological characteristics of a modified live porcine reproductive and respiratory syndrome virus (PRRSV) vaccine (ingelvac PRRS MLV), the parent strain of the vaccine (ATCC VR2332), ATCC VR2385, and two recent field isolates of PRRSV. J Virol 2002, 76, 11837-11844

25. Stadejek T, Porowski M, Pejsak Z. Viraemia and seroconversion in piglets following vaccination with PRRSV-EU type vaccine - a field observation. Bull Vet Inst Pulawy 2005, 49, 273-277.

26. Thanawongnuwech R, Amonsin A, Tatsanakit A, Damrong- 
watanapokin S. Genetics and geographical variation of porcine reproductive and respiratory syndrome virus (PRRSV) in Thailand. Vet Microbiol 2004, 101, 9-21.

27. van Woensel PAM, Leifkens K, Demaret S. Effect on viraemia of an American and a European serotype PRRSV vaccine after challenge with European wild-type strains of the virus. Vet Rec 1998, 142, 510-512.

28. Wensvoort G. Lelystad virus and the porcine epidemic abortion and respiratory syndrome. Vet Res 1993, 24, 117124.
29. Yin G, Gao L, Shu X, Yang G, Guo S, Li W. Genetic diversity of the ORF5 gene of porcine reproductive and respiratory syndrome virus isolates in southwest China from 2007 to 2009. PLoS One 2012, 7, e33756.

30. Yoo SJ, Kwon T, Kang K, Kim H, Kang SC, Richt JA, Lyoo YS. Genetic evolution of classical swine fever virus under immune environments conditioned by genotype 1-based modified live virus vaccine. Transbound Emerg Dis 2018, 65, 735-745. 